\title{
Microscopic Polyangiitis That Presented Liver Dysfunction Prior to Noted Renal Manifestations
}

\author{
Teruko Nakamoto, Masahide Yoshikawa*, Toshiya Nakatani, Yoshiko Yamane, Shu Iwasawa, \\ Madoka Matsumoto, Masaki Kawanami, Kimio Nishimura, Shigehiko UedA** and Hiroshi Fukui**
}

\begin{abstract}
In microscopic polyangiitis (MPA), renal manifestations are very common as first symptoms. Here, we report a case of MPA which presented liver dysfunction prior to noted renal manifestations. A 58-year-old woman was hospitalized because of a fever for 8 weeks. A laboratory examination revealed marked elevation of alkaline phosphatase and $\gamma$-glutamyl transpeptidase, while blood urea nitrogen and creatinine levels remained normal. Although apparent renal dysfunction developed in this case soon after hospitalization, physicians should be aware of the variety of clinical manifestations in MPA. Moreover, antineutrophil cytoplasmic autoantibodies were found to be helpful for diagnosing MPA.
\end{abstract}

(Internal Medicine 39: 517-521, 2000)

Key words: antineutrophil cytoplasmic autoantibodies (ANCA), small vessel vasculitis, crescentic glomerulonephritis, corticosteroid

\section{Introduction}

Small vessel vasculitis (SVV) is well known to be injurious to the kidney (1-5). Recently, anti-neutrophil cytoplasmic autoantibodies (ANCA) have been recognized as useful markers for diagnosing SVV and have been classified into two subsets by indirect immunofluorescent microscopy. One displays a perinuclear pattern (p-ANCA), which mainly reacts with myeloperoxidase (MPO), and the other displays a cytoplasmic staining pattern (c-ANCA), which reacts with a serine proteinase called proteinase 3 (PR3). Herein, we report a female patient with MPO-ANCA-positive SVV who presented liver dysfunction prior to the appearance of manifested renal dysfunction.

From the Department of Internal Medicine, Takanohara General Hospital, Nara, *the Department of Parasitology and **the Third Department of Internal Medicine, Nara Medical University, Kashihara

Received for publication July 27, 1999; Accepted for publication December 14, 1999

Reprint requests should be addressed to Dr. Masahide Yoshikawa, the Department of Parasitology, Nara Medical University, 840 Shijo-cho, Kashihara, Nara $634-8521$
For editorial comment, see $\mathrm{p} 449$.

\section{Case Report}

A 58-year-old woman visited our hospital on October 2, 1998, after enduring a fever for 8 weeks. She had no history of diseases or hospitalization, except for giving birth twice when in her twenties. Her height was $143 \mathrm{~cm}$ and her body weight was $42 \mathrm{~kg}$. Her body temperature was $38.3^{\circ} \mathrm{C}$, her pulse was regular at $98 / \mathrm{min}$, and her blood pressure was $140 / 80 \mathrm{mmHg}$. There was no rash or superficial lymphadenopathy. Her complexion was pallor and her sclera was not icteric. Findings on examination of her heart and lungs were normal, and there was no tenderness in the abdomen. The liver, gall bladder, spleen, and kidneys could not be felt. There was no peripheral edema, and neither arthralgia nor peripheral neuropathy were observed. The results of her laboratory tests are shown in Table 1. Urine was trace positive for protein and blood. The sediment contained 5-9 red blood cells, when viewed by high-power microscopy. A feces sample was negative for occult blood. A blood examination disclosed leucocytosis with neutrophil prevalence (white blood cells 11,500/ $\mu 1,86.6 \%$ neutrophils), elevated Creactive protein (CRP, $13.5 \mathrm{mg} / \mathrm{dl})$, and liver dysfunction including alkaline phosphatase (ALP) $996 \mathrm{U} / l$ and $\gamma$-glutamyl transpeptidase ( $\gamma$-GTP) $274 \mathrm{U} / l$. The blood urea nitrogen (BUN) and creatinine (Cr) levels were both normal, while the antinuclear antibody (ANA) was highly positive and IgG was increased. The levels of complement C3, C4, CH50, and IgM were all within normal ranges. The anti-mitochondrial antibody (AMA) was not detected. All of examinations including abdominal ultrasonography (US), chest X-P, abdominopelvic computed tomography (CT), and endoscopic retrograde cholangiopancreatography found no abnormalities. Before visiting us, she had consulted two neighborhood physicians on August 29 and September 21, 1998, and was treated with a non-steroidal anti-inflammatory drug (NSAID) for 2 days by 
NAKAMOTo et al

Table 1. Laboratory Data on Admission

\begin{tabular}{|c|c|c|c|c|c|c|c|}
\hline Urinalysis & & $\mathrm{RBC}$ & $413 \times 10^{4} / \mu \mathrm{I}$ & BUN & $15 \mathrm{mg} / \mathrm{dl}$ & anti-RNP & 4.3 \\
\hline Protein & $( \pm)$ & $\mathrm{Hb}$ & $11.1 \mathrm{~g} / \mathrm{dl}$ & $\mathrm{Cr}$ & $1.0 \mathrm{mg} / \mathrm{dl}$ & anti-Sm & 1.8 \\
\hline Occult blood & $( \pm)$ & $\mathrm{Ht}$ & $34.6 \%$ & $\mathrm{Na}$ & $136 \mathrm{mEq} / \mathrm{l}$ & \multicolumn{2}{|c|}{ anti-CL- $\beta_{2} \mathrm{GP} 1<1.3 \mathrm{U} / \mathrm{ml}$} \\
\hline Sugar & $(2+)$ & Plt & $50.7 \times 10^{4} / \mu 1$ & $\mathrm{~K}$ & $4.1 \mathrm{mEq} / \mathrm{l}$ & $\mathrm{C} 3$ & $88 \mathrm{mg} / \mathrm{dl}$ \\
\hline Sediment & & ESR & $147 \mathrm{~mm} / \mathrm{hr}$ & $\mathrm{Cl}$ & $92 \mathrm{mEq} / \mathrm{l}$ & $\mathrm{C} 4$ & $26.9 \mathrm{mg} / \mathrm{dl}$ \\
\hline RBC & $5-9 / \mathrm{HPF}$ & & & CRP & $13.5 \mathrm{mg} / \mathrm{dl}$ & CH50 & $40 \mathrm{U} / \mathrm{ml}$ \\
\hline WBC & $1-4 / \mathrm{HPF}$ & \multicolumn{2}{|c|}{ Blood chemistry } & Glu & $120 \mathrm{mg} / \mathrm{dl}$ & \multicolumn{2}{|c|}{ anti-thyroglobulin $<\times 100$} \\
\hline Epithel & $1-4 / \mathrm{HPF}$ & TP & $7.7 \mathrm{~g} / \mathrm{dl}$ & & & anti-microsom & e $<\times 100$ \\
\hline \multirow[t]{2}{*}{ no casts } & & Alb & $3.1 \mathrm{~g} / \mathrm{dl}$ & Serology & & HBsAg & \\
\hline & & T-Bil & $0.5 \mathrm{mg} / \mathrm{dl}$ & $\mathrm{IgG}$ & $2,650 \mathrm{mg} / \mathrm{dl}$ & anti-HCV3rd & $(-)$ \\
\hline Hematology & & TTT & $1.9 \mathrm{U}$ & IgA & $364 \mathrm{mg} / \mathrm{dl}$ & & \\
\hline WBC & $11,500 / \mu \mathrm{l}$ & ZTT & $10.5 \mathrm{U}$ & $\operatorname{IgM}$ & $10 \mathrm{mg} / \mathrm{dl}$ & \multicolumn{2}{|l|}{ Tumor marker } \\
\hline Neutr & $86.6 \%$ & AST & $21 \mathrm{IU} / l$ & IgE & $86 \mathrm{IU} / \mathrm{ml}$ & CEA & $<0.9 \mathrm{ng} / \mathrm{ml}$ \\
\hline Lym & $7.3 \%$ & ALT & $19 \mathrm{IU} / l$ & $\gamma-\mathrm{gl}$ & $2.05 \mathrm{~g} / \mathrm{dl}$ & CA19-9 & $<10 \mathrm{ng} / \mathrm{ml}$ \\
\hline Mono & $4.0 \%$ & ALP & $996 \mathrm{U} / l$ & $\mathrm{RF}$ & $18 \mathrm{U} / \mathrm{ml}$ & AFP & $1.8 \mathrm{ng} / \mathrm{ml}$ \\
\hline Eosino & $1.1 \%$ & $\gamma$-GTP & $274 \mathrm{U} / \mathrm{l}$ & ANA(speckled) & d) $\times 2,560$ & & \\
\hline \multirow[t]{3}{*}{ Baso } & $0.9 \%$ & LAP & $95 \mathrm{U} / \mathrm{l}$ & anti-DNA & $4.2 \mathrm{IU} / \mathrm{ml}$ & & \\
\hline & & $\mathrm{T}-\mathrm{CHO}$ & $71 \mathrm{mg} / \mathrm{dl}$ & LE test & negative & & \\
\hline & & TG & $158 \mathrm{mg} / \mathrm{dl}$ & AMA & $<\times 10$ & & \\
\hline
\end{tabular}

Normal ranges: AST 10-40, ALT 5-45, ALP 74-223, $\gamma$-GTP<60, LAP 30-78.

Table 2. Laboratory Data before Admission

\begin{tabular}{|c|c|c|c|c|c|}
\hline & 29 Aug, & $21 \mathrm{Sep}$ & & 29 Aug, & $21 \mathrm{Sep}$ \\
\hline \multicolumn{3}{|l|}{ Urinalysis } & \multicolumn{3}{|l|}{ Blood chemistry } \\
\hline Protein & $(-)$ & $( \pm)$ & (g/dl) & 7.0 & \\
\hline Occult blood & $( \pm)$ & $(-)$ & $(\mathrm{IU} / l)$ & 26 & 18 \\
\hline \multirow[t]{2}{*}{ Sugar } & $(-)$ & $(-)$ & $(\mathrm{IU} / l)$ & 25 & 18 \\
\hline & & & $(\mathrm{IU} / l)$ & 420 & \\
\hline Hematology & & & $\gamma$-GTP $\quad(\mathrm{U} / l)$ & 124 & \\
\hline WBC $\quad(/ \mu l)$ & 7,300 & 10,400 & BUN $\quad(\mathrm{mg} / \mathrm{dl})$ & 11 & 10 \\
\hline $\mathrm{RBC}\left(\times 10^{4} / \mu \mathrm{l}\right)$ & 444 & 422 & $(\mathrm{mg} / \mathrm{dl})$ & 1.0 & 0.9 \\
\hline$(\mathrm{g} / \mathrm{dl})$ & 12.4 & 11.5 & $\beta_{2} \mathrm{MG} \quad(\mathrm{mg} / l)$ & 1.3 & \\
\hline$(\%)$ & 38 & 36 & CRP (mg/dl) & & 8.5 \\
\hline Plt $\quad\left(\times 10^{4} / \mu 1\right)$ & 35.1 & 31.3 & & & \\
\hline
\end{tabular}

Normal range: $\beta_{2} \mathrm{MG}\left(\beta_{2}\right.$-microglobulin) 0.8-2.4.

the first doctor and with antibiotics and another NSAID for 3 days by the second. Almost no effect was observed. Laboratory data obtained on August 29 and September 21 are shown in Table 2. Elevated ALP and $\gamma$-GTP were already present on August 29. A lymphocyte stimulation test, performed after admission using these NSAIDs and antibiotics, did not give positive results, suggesting it unlikely that her abnormal liver function was due to the use of these drugs.

A definite response to a 5-day treatment with antibiotics or the detection of pathogenic organisms in cultures from urine and blood could not be obtained, while a worsening of her renal functions gradually appeared, along with apparent proteinuria and microscopic hematuria, causing us to suspect systemic vasculitis rather than infection. Therefore, we performed an ANCA checkup. The BUN and Cr levels had reached 40 $\mathrm{mg} / \mathrm{dl}$ and $2.6 \mathrm{mg} / \mathrm{dl}$, respectively, on October 13, when we learned that MPO-ANCA was positive at 236 enzyme-linked immunoassay units (EU). We made a diagnosis of microscopic polyangiitis (MPA), a type of ANCA-associated vasculitis, according to the recent international nomenclature definitions of systemic vasculitis (5). Simultaneously, we introduced corticosteroid therapy, initiating intravenous administration of methylprednisolone $1.0 \mathrm{~g} /$ day for 3 days followed by an oral administration of prednisolone, starting at a daily dose of 40 mg. PR3-ANCA was negative. After reaching this diagnosis, we again attempted to discover any manifestations, even subclinical, related to organs other than the kidneys and liver including lungs, skin, muscle, gastrointestinal tract and eyes. 


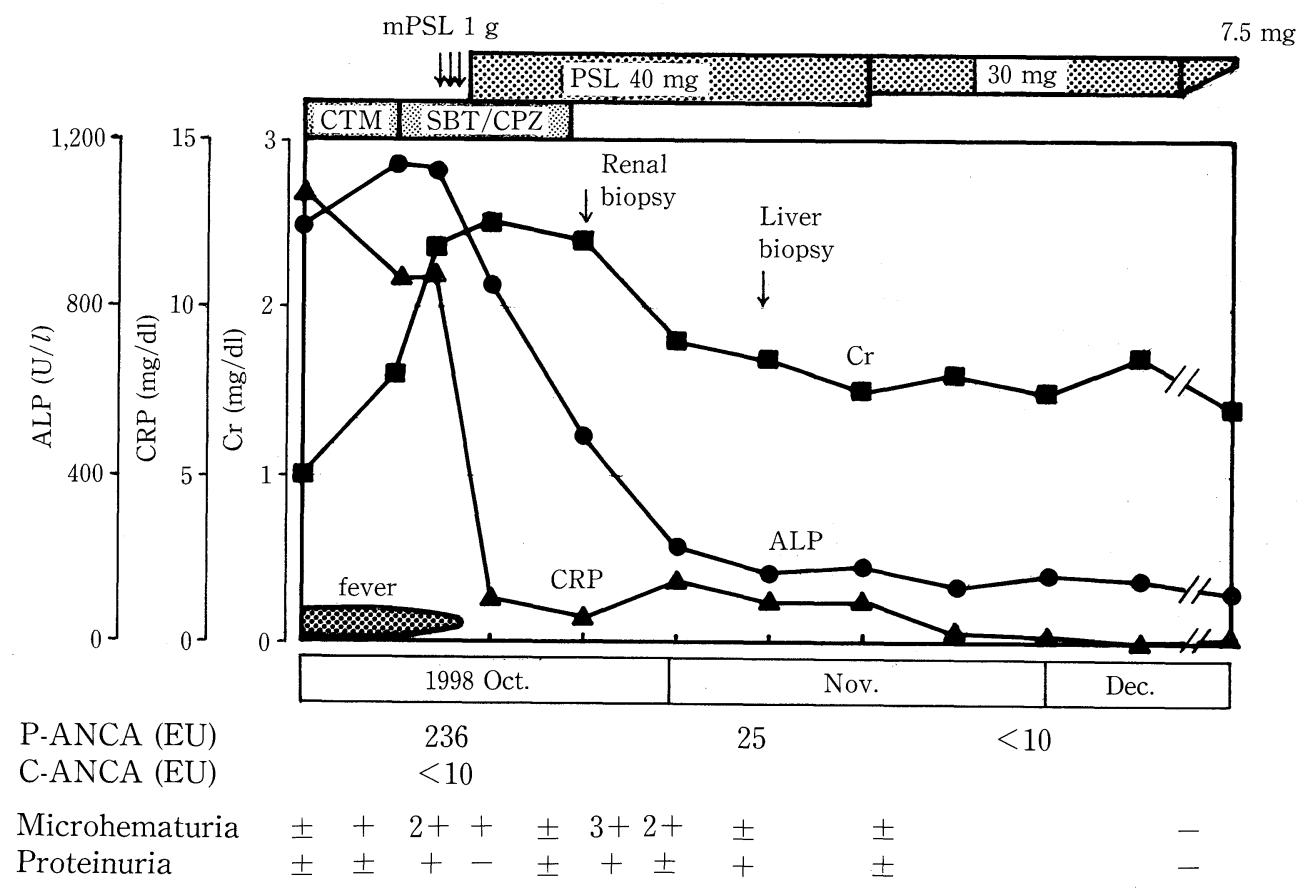

Figure 1. Clinical course and laboratory data of the patient. mPSL: methyl-prednisolone, PSL: prednisolone, CTM: Cefotiam, SBT/CPZ: sulbactam/cefoperazone.

However, no abnormalities were found. In addition, peripheral nerves in extremities showed normal conduction verocity.

Prompt improvement in clinical symptoms and laboratory findings was obtained after the start of corticosteroid therapy (Fig. 1). ALP and $\gamma$-GTP values were normalized within two weeks, but her $\mathrm{Cr}$ levels remained at around $1.5 \mathrm{mg} / \mathrm{dl}$. A USguided needle biopsy was performed from the right kidney on October 23. The specimen contained 16 glomeruli, of which one glomerulus was almost completely destroyed and in the process of being replaced by fibrosis (Fig. 2, right), while eight glomeruli presented segmental fibrinoid necrosis. Six out of the eight showed focally fractured Bowman's capsules, and two of them were accompanied by a small cellular crescent formation. Infiltration of inflammatory cells was observed in the interstitium surrounding the destroyed Bowman's capsules (Fig. 2, left). Necrotizing inflammation involving interlobular arteries was not found. Deposition of immunoglobulin and complements was not detected by immunohistologic staining. On November 4, we performed a percutaneous needle biopsy from the right lobe of the liver. Although few inflammatory infiltrates were observed in the portal tracts, intramural fibrinoid degeneration was found in an interlobular arteriole (Fig. 3). Moreover, the epithelial cells of the bile duct adjacent to the affected arteriole seemed to be disarranged.

Remission was achieved while prednisolone was gradually being tapered and maintained at a daily dose of $7.5 \mathrm{mg}$. The CRP levels did not exceed $1.0 \mathrm{mg} / \mathrm{dl}$ and hematuria was controlled at negative or trace positive by a tape method, although a complete normalization of serum was not obtained. The patient is now under maintenance therapy with prednisolone ( 7.5 $\mathrm{mg} /$ day) and dipyridamole (300 mg/day).

\section{Discussion}

In 1993, the Chapel Hill Consensus Conference for the Nomenclature of Systemic Vasculitis agreed on the names and definitions of many vasculitides that affect the kidneys (5). Based on the definitions determined in the international consensus, we diagnosed the present patient as having MPA. However, we could not reach this diagnosis before the development of apparent renal manifestations such as hematuria, proteinuria, and elevated $\mathrm{Cr}$ levels. The most frequent clinical features in systemic vasculitis, especially in SVV, are renal manifestations, although they may vary according to the severity and stage of the underlying renal injury. In the present case, neither proteinuria nor hematuria was apparent at the beginning of hospitalization, which led us to consider systemic vasculitis as unlikely. We had believed the possible diagnosis to be infection, malignancy, drugs-induced liver disease, or those complicated with some biliary tract disease such as primary biliary cirrhosis, primary sclerosing cholangitis, or common bile duct stones.

The presence of ANCA was helpful for diagnosing systemic vasculitis. ANCA-positive SVV (ANCA-SVV) is divided into three major clinicopathologic categories; MPA, Wegener's granuloma, and Churg-Strauss syndrome (3-5). They share 

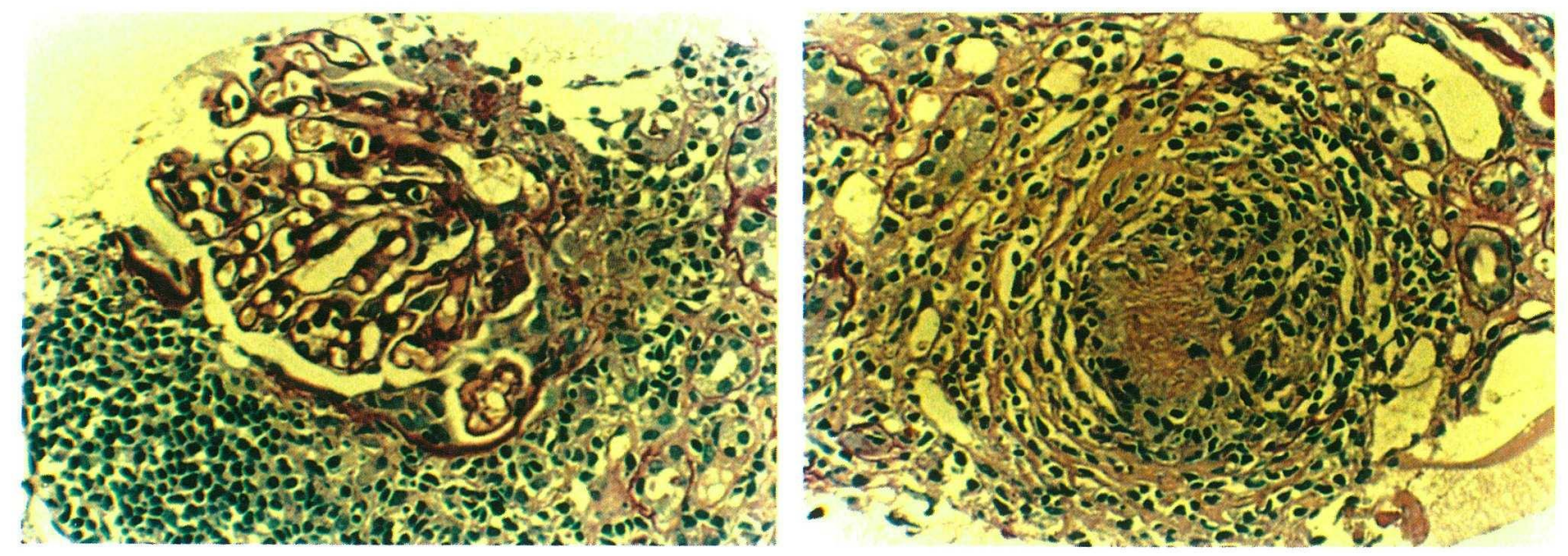

Figure 2. Light microscopic findings of the renal biopsy specimens (PAS stain, $\times 400)$. Left: Cellular crescent formation in Bowman's capsules and marked infiltration of inflammatory cells in the interstitium. Right : A glomerulus which is almost completely destroyed and being replaced by fibrosis.

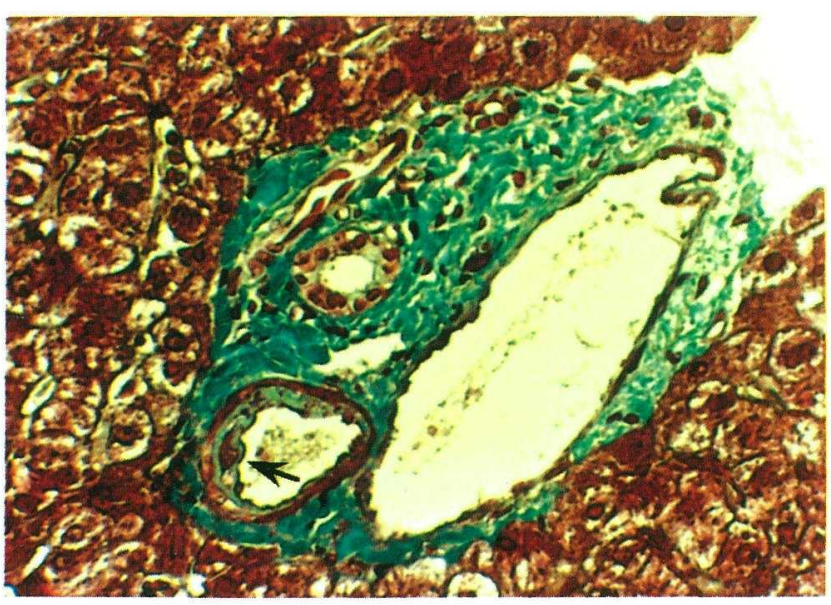

Figure 3. Light microscopic findings of the liver biopsy specimen. Fibrinoid degeneration in the wall of an interlobular arteriole (arrow) was found (Azan stain, $\times 400$ ).

pathologically identical necrotizing inflammation in the small vessels. Wegener's granuloma is distinguished by the presence of necrotizing granulomatous inflammation involving the respiratory tract, Churg-Strauss syndrome by the presence of asthma and eosinophilia, and MPA by the absence of granulomatous inflammation, eosinophilia, or asthma, as was seen in the present case.

The kidneys are the most often affected organ in the majority of patients with MPA and renal manifestations are usually the first symptoms. Glomerular capillaries are affected most often, resulting in necrotizing glomerulonephritis, usually in a crescent formation, with no or few immune deposits able to be proven at the sites of vasculitis and glomerulonephritis, all of which are consistent with the present case. A recent study ana- lyzing 85 patients with MPA (6) demonstrated that renal manifestations were the most frequent, being present in 67 patients (78.8\%), as well as fever and weight loss. However, other manifestations related to skin (62.4\%), nerve (57.6\%), joint (50.6\%), muscle $(48.2 \%)$, gastrointestinal tract $(30.6 \%)$, and lung $(24.7 \%)$ have also been observed. Therefore, it should be emphasized that a wide variety of clinical manifestations may exist due to the varying tissue distribution of ANCA-SVV inflammatory lesions among patients.

According to the same study, 10 patients (11.8\%) were reported to show abnormal findings in liver function tests, such as an elevation of aspartate aminotransferase (AST) and/or alanine aminotransferase (ALT) levels. However, the liver dysfunction seen in the present case was an elevation of ALP and $\gamma$-GTP, not AST or ALT. Furthermore, the appearance of liver dysfunction was prior to the noted renal manifestations. Recently, a case of MPA similar to our patient was reported (7), in whom liver dysfunction consisting of an elevation of ALP and $\gamma$-GTP developed before noted renal manifestations. Liver biopsies from that patient revealed necrotizing arteritis, infiltration of inflammatory cells to the portal tract surrounding the affected artery, and subsequent fibrosis of the portal tract. In the present case, however, only a few inflammatory infiltrates were observed in the portal area and no hepatic artery branch was found in the liver needle-biopsy specimens that displayed active necrotizing lesions. Even though almost no findings demonstrating arteritis in the liver specimens were observed, a small intramural fibrinoid degeneration was found in an interlobular arteriole, suggesting preceding arteriolitis in the liver. Irregularly arranged epithelial cells of the adjacent bile duct also suggested involvement of the bile duct in the inflammatory process. Considering that a 3 -week treatment of prednisolone preceded the liver biopsy, it is possible that active inflammation was inhibited by the anti-inflammatory effects of corticosteroid prior to the liver biopsy. We believe that prominent, 
but not severe, arteritis existed in the liver before the institution of corticosteroid.

It is known that angiograms of affected organs are often helpful to detect medium-sized vessel involvement in patients with systemic vasculitis (8). Microaneurysms and/or multiple vessel stenosis reflect medium-sized vessel involvement. Although MPA is defined by necrotizing vasculitis affecting small vessels, medium-sized vessel vasculitis may be present (5). We should have considered an angiogram of the liver when we suspected systemic vasculitis. Microaneurysms and/or multiple vessel stenosis, when present on hepatic arteriograms, suggest the presence of arteritis in the liver. However, the frequency of abnormal angiograms has been reported to be very low in patients with ANCA-SVV (8).

To suppress the vascular inflammation promptly, we administered high-dose corticosteroid ( $1 \mathrm{~g} /$ day of methylprednisolone) initially by pulse intravenous therapy on three consecutive days, followed by daily oral dosing of $40 \mathrm{mg}$ (about $1 \mathrm{mg} /$ $\mathrm{kg}$ body weight), tapered to $7.5 \mathrm{mg}$ over 4 months and then maintained at that dose. Cyclophosphamide can also be used either intravenously or orally in combination with corticosteroid as a drug of induction therapy $(3,4)$. In the present patient, improvement of renal function along with resolution of hematuria was obtained by the corticosteroid mono-therapy. The patient is now under a maintenance therapy with prednisolone ( $7.5 \mathrm{mg} /$ day) and dipyridamole $(300 \mathrm{mg} /$ day).

According to studies examining prognostic factors in SVV $(6,9,10)$, the following items were considered to be attributable; (a) the entry-level serum creatinine value, (b) the severity of renal disease, (c) proteinuria, (d) the presence of pulmonary disease, (e) the presence of cardiomyopathy, (f) the presence of gastrointestinal tract involvement, (g) the presence of central nervous system involvement, and (h) the use of cyclophosphamide in combination with corticosteroid. The entrylevel serum creatinine levels are largely attributed to the severity of renal disease. Among factors (a) to (h), Falk and Jennette stated in a review article that the most important determinant of patient survival is the presence or absence of pulmonary hemorrhage (4). We recently lost another patient with MPA due to this complication (11). Some French investigators $(6,9)$ have advocated the five-factors score (FFS), a scoring system used to predict the outcome of patients with MPA, which comprises (a) creatinine level $>1.58 \mathrm{mg} / \mathrm{dl}$, (c) proteinuria $>1 \mathrm{~g} /$ day, (e), (f), and (g). According to the FFS, our patient scored 1 point because a high level of creatinine $(2.6 \mathrm{mg} / \mathrm{dl}$ at the start of corticosteroid therapy) was present, thus, we are expecting an approximately $85 \%$ chance of 5 -year survival (6).

Until the present time, no signs of relapse, such as those listed by Nachman et al (12), have been observed. However, it has been reported that the risk of relapse in mono-therapy using corticosteroid is three-fold higher as compared to a combination therapy of corticosteroid and cyclophosphamide $(3,4$, 12). In addition to careful observation for clinical manifestations, we believe that monitoring of the ANCA titers is important and useful for the early detection of relapse in the present case. Since the ANCA titers gradually decreased and finally became negative by corticosteroid therapy in this patient, they seem to be closely correlated to the disease activity, as other investigators have observed (13-15).

In summary, we report a case of ANCA-associated vasculitis that presented liver dysfunction prior to noted renal manifestations. Although apparent renal dysfunction developed soon after hospitalization in the present case, physicians should be aware of the variety of clinical manifestations accompanying MPA.

\section{References}

1) Falk RJ, Jennette JC. Anti-neutrophil cytoplasmic autoantibodies with specificity for myeloperoxidase in patients with systemic vasculitis and idiopathic necrotizing and crescentic glomerulonephritis. N Engl J Med 318: 1651-1657, 1988.

2) Hagen EC, Ballieux BE, van Es LA, Daha MR, van der Woude FJ. Antineutrophil cytoplasmic autoantibodies: a review of the antigens involved, the assays, and the clinical and possible pathogenetic consequences. Blood 81: 1996-2002, 1993.

3) Jennette JC, Falk RJ. Small-vessel vasculitis. N Engl J Med 337: 15121523, 1997.

4) Falk RJ, Jennette JC. ANCA small-vessel vasculitis. J Am Soc Nephrol 8: 314-322, 1997.

5) Jennette JC, Falk RJ, Andrassy K, et al. Nomenclature of systemic vasculitides. Proposal of an international consensus conference. Arthritis Rheum 37: 187-192, 1994.

6) Guxillevin L, Durand-Gasselin B, Cevallos R, et al. Microscopic polyangiitis: clinical and laboratory findings in eighty-five patients. Arthritis Rheum 42: 421-430, 1999.

7) Goritsas CP, Repanti M, Papadaki E, Lazarou N, Andonopoulos AP. Intrahepatic bile duct injury and nodular regenerative hyperplasia of the liver in a patient with polyarteritis nodosa. J Hepatol 26: 727-730, 1997.

8) Guillevin L, Lhote F, Amouroux J, Gherardi R, Callard P, Casassus P. Antineutrophil cytoplasmic antibodies, abnormal angiograms and pathological findings in polyarteritis nodosa and Churg-Strauss syndrome: indications for the classification of vasculitides of the polyarteritis Nodosa Group. Br J Rheumatol 35: 958-964, 1996.

9) Guillevin L, Lhote F, Gayraud M, et al. Prognostic factors in polyarteritis nodosa and Churg-Strauss syndrome. A prospective study in 342 patients. Medicine (Baltimore) 75: 17-28,1996.

10) Hogan SL, Nachman PH, Wilkman AS, Jennette JC, Falk RJ. Prognostic markers in patients with antineutrophil cytoplasmic autoantibody-associated microscopic polyangiitis and glomerulonephritis. J Am Soc Nephrol 7: 23-32, 1996.

11) Ueda S, Matsumoto M, Ann T, et al. A case of fatal microscopic polyangiitis that presented with massive intestinal bleeding and pulmonary hemorrhage (Submitted).

12) Nachman PH, Hogan SL, Jennette JC, Falk RJ. Treatment response and relapse in antineutrophil cytoplasmic autoantibody-associated microscopic polyangiitis and glomerulonephritis. J Am Soc Nephrol 7: 33-39, 1996.

13) Jayne DR, Gaskin G, Pusey CD, Lockwood CM. ANCA and predicting relapse in systemic vasculitis. QJM 88: 127-133, 1995.

14) Geffriaud-Ricouard C, Noel LH, Chauveau D, Houhou S, Grunfeld JP, Lesavre P. Clinical spectrum associated with ANCA of defined antigen specificities in 98 selected patients. Clin Nephrol 39: 125-136, 1993.

15) Fujimoto T, Kimura A, Ameno M, Shiiki H, Dohi K. A case of necrotizing crescentic glomerulonephritis in which myeloperoxidase antineutrophil cytoplasmic antibodies (ANCA) reflect the disease activity and recognize various neutrophil cytoplasmic constituents. Nippon Jinzo Gakkai Shi (Jpn J Nephrol) 39: 172-177, 1997 (Abstract in English). 